\title{
Geotail Observations of an Unusual Magnetotail under Very Northward IMF Conditions
}

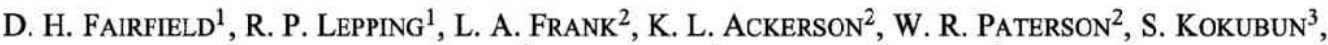 \\ T. YAMAMOTO ${ }^{4}, \mathrm{~K}$. TSURUdA ${ }^{4}$, and M. NAKAMURA ${ }^{5}$ \\ ${ }^{1}$ Laboratory for Extraterrestrial Physics, NASA Goddard Space Flight Center, Greenbelt, Maryland, U.S.A. \\ ${ }^{2}$ Department of Physics and Astronomy, University of Iowa, Iowa City 52242, U.S.A. \\ ${ }^{3}$ STELAB, Nagoya University, Toyokawa 442, Japan \\ ${ }^{4}$ ISAS, Sagamihara 229, Japan \\ ${ }^{5}$ Dept. of Earth and Planetary Physics, The University of Tokyo, Hongo 7-3-l, Bunkyou-ku, Tokyo 113, Japan
}

(Received October 13, 1995; Accepted January 27, 1996)

On March 29, 1993, the Geotail spacecraft was $134 R_{E}$ downstream from the earth and within $12 R_{E}$ of the axis of the magnetotail whose orientation was calculated from the solar wind direction measured by IMP 8 . The IMF had a magnitude near $12 \mathrm{nT}$ and remained steady and roughly $50^{\circ}$ northward for 9 hours. Geotail moved back and forth between two regions, an interior region with characteristics similar to the tail and an exterior region with characteristics similar to the magnetosheath. The flow in the dense rapidly flowing exterior region was tilted $8^{\circ}$ toward the tail axis rather than parallel to a cylindrical tail. Calculated boundary normals were primarily in the solar magnetospheric $Y$ direction but with a small $X$ component consistent with a slightly flaring or cylindrical tail. The unusual flow direction in the exterior region along with the presence of slower, less dense, tailward flowing plasma on taillike field lines inside the boundary suggest that the exterior flow has a component across the boundary and into the tail. These data support accumulating evidence from MHD simulations that suggest that the magnetotail assumes a very different configuration when the IMF remains very northward for periods of at least several hours. In this unusual configuration, field lines which would normally extend into the distant tail instead close on the flanks and nearer to the earth, creating a distant tail with less open flux and a reduced $Y$ dimension.

\section{Introduction}

Recent simulations (Usadi et al., 1993; Ogino et al., 1994; Fedder and Lyon, 1995; Raeder et al., 1995) and observations (Fairfield, 1993) indicate that the magnetotail assumes a very different configuration when the IMF (Interplanetary Magnetic Field) maintains an extremely northward orientation for at least several hours. Simulations support early suggestions (Song and Russell (1992) and references therein) whereby high latitude reconnection between northward IMF field lines and tail field lines poleward of the dayside cusps create dayside field lines containing magnetosheath plasma that are closed, or at least attached to the earth at one end, and whose direction resembles that of magnetosphere field lines. Such field lines are expected to convect around the earth toward the night side where they either enhance the closed flux in the tail if they are closed or at least resemble closed tail field lines even if they are not closed. The simulations indicate that within several hours the tail assumes a state where virtually all field lines are closed (Fedder and Lyon, 1995) or at least a significantly increased number of field lines are closed (Ogino et al., 1992, 1994; Usadi et al., 1993; Raeder et al., 1995) so that the distant tail has a reduced $Y$ dimension. Either case is very different from the typical long tail of open field lines whose average radius is $24 \mathrm{R}_{\mathrm{E}}$ at a distance of $200 \mathrm{R}_{\mathrm{E}}$ behind the earth (Fairfield, 1992).

In this paper we investigate a period when the Geotail spacecraft was $134 R_{E}$ behind the earth and observing an anomalous tail during a period when the IMF was unusually strong and steady and generally $45-55^{\circ}$ northward for about 9 hours. We concentrate on high resolution magnetic field and plasma data in an attempt to elucidate the interaction processes under these uniquely steady and northward conditions. 


\section{Observations}

During the last half of March 29, 1993, the IMP 8 spacecraft measured a solar wind with nearly constant velocity of $370 \mathrm{~km} / \mathrm{s}$ and a density that increased gradually from near $8 / \mathrm{cc}$ to about $20 / \mathrm{cc}$. The IMF increased from 11 to $14 \mathrm{nT}$ and the direction was unusually steady and about $45-55^{\circ}$ northward between 1200 and 2100 UT. The direction of the (unaberrated) solar wind was 2.5 degrees from the east of the sun (dawnward) and 2.6 degrees northward after a $1.8^{\circ}$ instrumental bias was subtracted. IMP 8 was at a location near $X=34, Z=-15$ with $Y$ changing from -16 to $-7 \mathrm{R}_{\mathrm{E}}$ during this period.

During this same time interval the Geotail spacecraft was $134 \mathrm{R}_{\mathrm{E}}$ down the tail moving from a solar ecliptic $X Y Z$ location $(-134.5,14.4,-.7)$ to $(-132.5,13.9,-9)$. The aberration of the tail for a $370 \mathrm{~km} / \mathrm{s}$ solar wind would rotate a nominal tail $4.6^{\circ}$ toward dusk, but the actual solar wind average direction of $2.5^{\circ}$ toward dawn would give a net displacement of the tail of only $2.1^{\circ}$ toward dusk. This displacement in effect reduces the $Y$ coordinate of Geotail by $4.9 \mathrm{R}_{\mathrm{E}}$. The $2.6^{\circ}$ northward flow to the solar wind raises the tail by $6.0 \mathrm{R}_{\mathrm{E}}$. This displacement of the tail means that on average Geotail has a position relative to the tail center

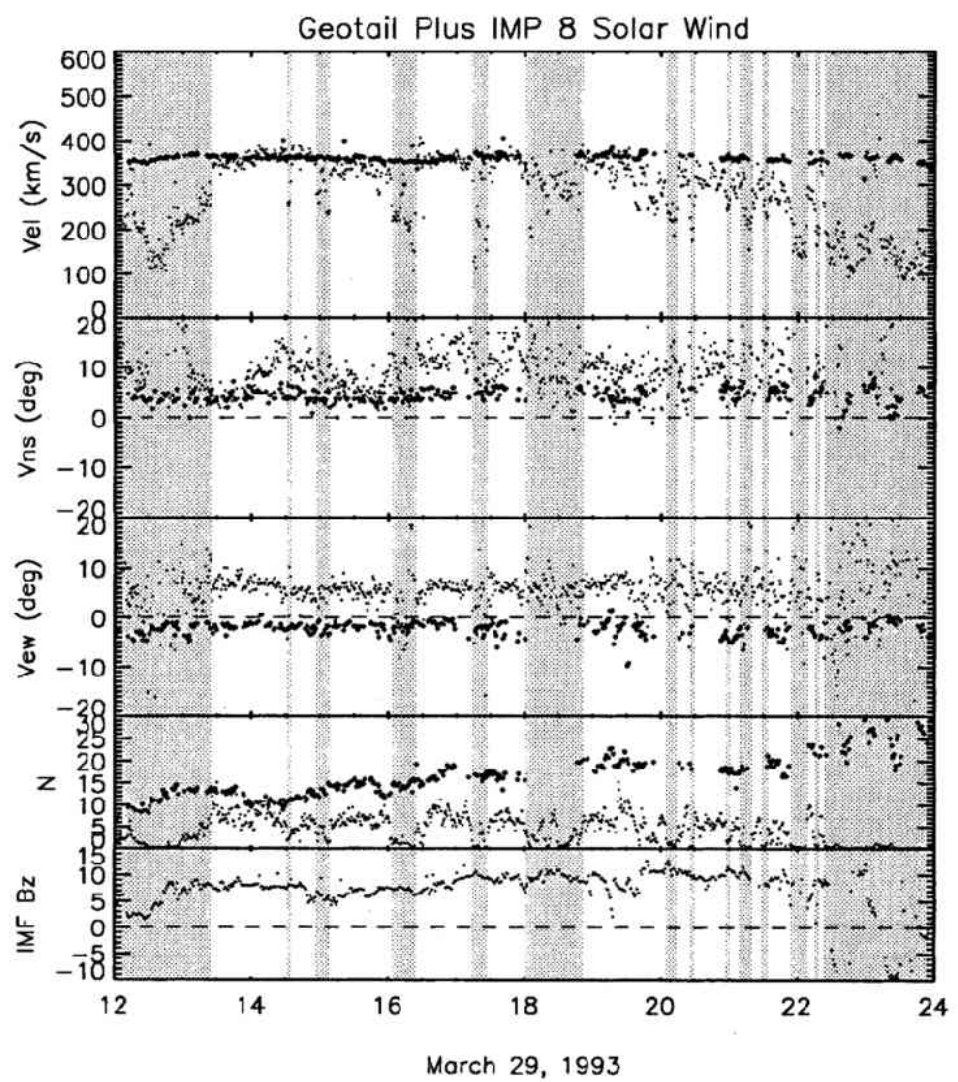

Fig. Ia. Solar wind parameters (heavy points) compared with those of Geotail (light points) for a 12 hour interval on March 29 , 1993. The various panels represent the plasma speed and north/south and east/west directions (northward and dawnward are positive), density and IMF north/south component. Shading indicates regions of low density and velocity where Geotail has entered a region associated with the distant tail. Solar wind quantities have been advanced 48 minutes to account for the Imp $8 /$ Geotail transit time. 
of $Y=9.3 \mathrm{R}_{\mathrm{E}}, Z=-6.8 \mathrm{R}_{\mathrm{E}}$ (i.e., $11.5 \mathrm{R}_{\mathrm{E}}$ from the tail center). Before about 1600 hours UT the IMF has a positive $B_{y}$ component which should rotate the tail counterclockwise, looking down the tail from the earth (Cowley, 1981; Sibeck et al., 1986; Owen et al., 1995; Nishida et al., 1995), which will tend to move the southern half of the tail northward at the Geotail location on the dusk flank. At later times the IMF $B_{y}$ component becomes negative and the rotation will be reversed. These rotations are consistent with the Geotail observations as will be seen below.

Figures $1 \mathrm{a}$ and $\mathrm{lb}$ compares the plasma and magnetic field measurements respectively from the two spacecraft for a 12 hour period on March 29, 1993. In Fig. 1a the heavy dots represent IMP 8 and the lighter points Geotail. The IMP 8 data have been advanced in time by 48 minutes to account for the convection time from IMP 8 to $134 \mathrm{R}_{\mathrm{E}}$. The plasma velocity comparisons in the top panel of Fig. 1a indicate very similar speeds at the two spacecraft during some intervals, but large decreases at Geotail during other intervals which are indicated by shading. Geotail density measurements in the fourth panel tend to be about half the solar wind densities in the unshaded region but are significantly lower in the shaded region. Although the Geotail plasma data of Fig. 1a are obtained by the solar wind portion of the CPI (Comprehensive Plasma Instrument) which is the most appropriate instrument for measuring the dense,

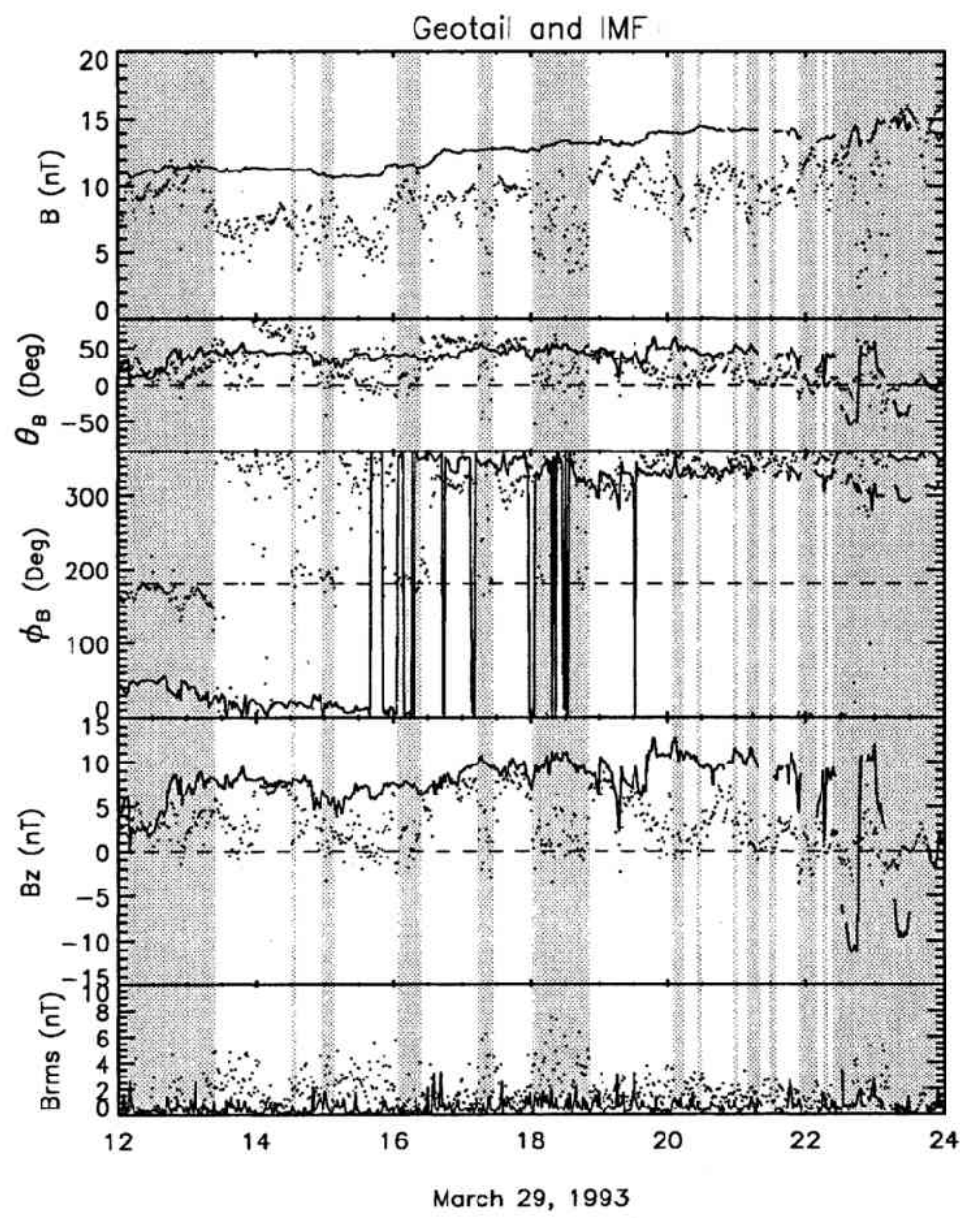

Fig. 1b. IMF magnitude and latitude and longitude angles and $B_{z}$ component represented by the solid line are compared to simultaneously measured quantities on Geotail. Shading is as in Fig. 1a and the IMF is advanced by the same 48 minutes. 
cool plasmas of the solar wind or magnetosheath, data from the CPI hot plasma analyzer shown in later figures will confirm the lower density, more isotropic, taillike plasmas in the shaded regions.

The lines and points in Fig. 1b illustrate the magnetic field data from IMP 8 and Geotail respectively. The field latitude and longitude angles in solar magnetospheric coordinates, $\theta$ and $\phi$ indicate similar directions in the unshaded regions but often different directions in the low density, low velocity shaded regions. Before 1830 the Geotail field directions in the shaded regions tend to be near $180^{\circ}$ characteristic of the southern tail which would be expected for a tail displaced northward and rotated in response to an IMF with positive $B_{y}$ (Cowley, 1981; Sibeck et al., 1986; Owen et al., 1995; Nishida et al., 1995). In the unshaded region the Geotail field direction tends to be nearer $0^{\circ}$ or $360^{\circ}$ which is similar to the IMF. After

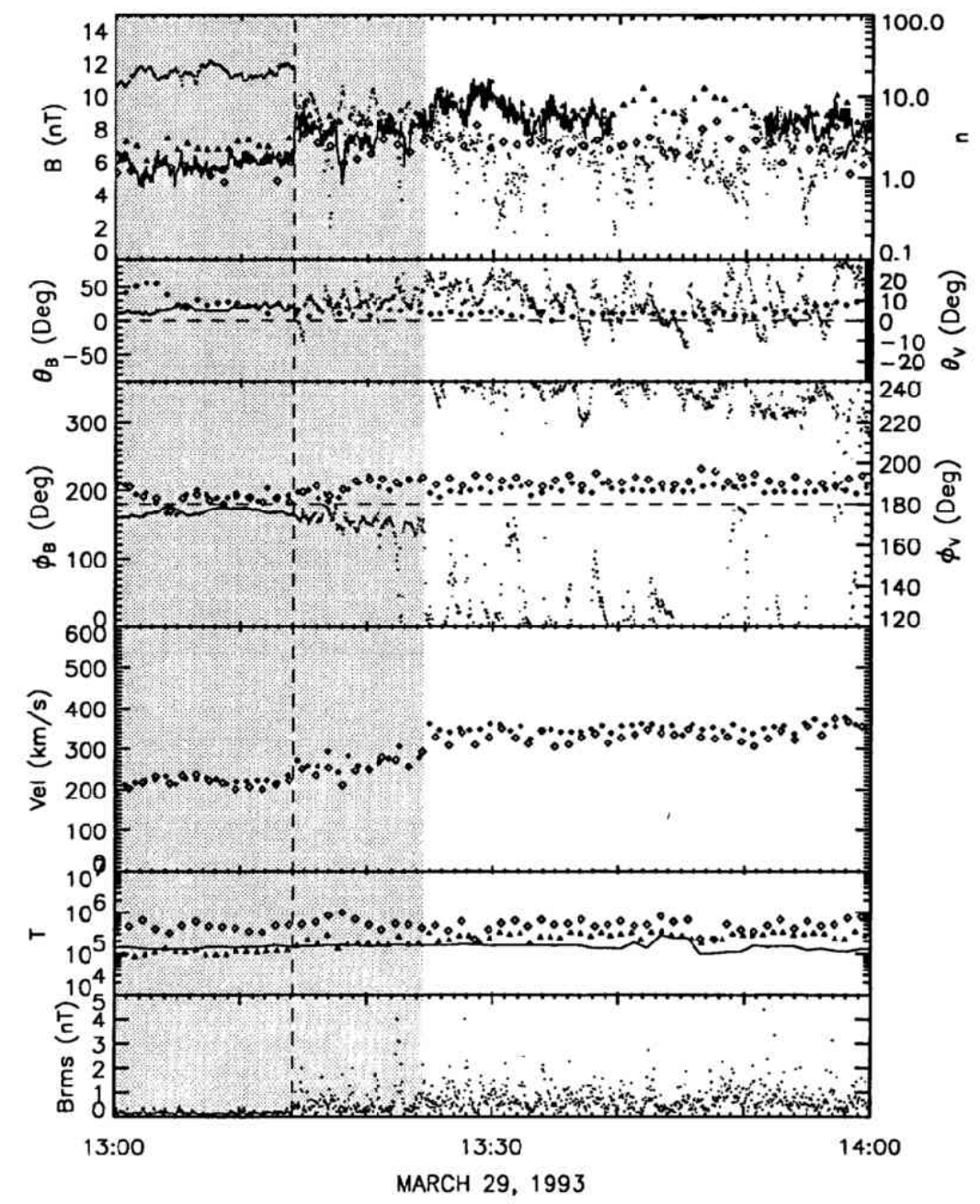

Fig. 2a. The top panel contains one hour of Geotail magnetic field magnitude (light points) along with densities from the CPI hot plasma (open diamonds) and solar wind (solid triangles) detectors. Density estimated from the EFD potential measurement is indicated by the solid line. Latitude and longitude angles for the magnetic field and plasma flow are shown in the second and third panels (note the different scales). Velocity is shown in the fourth pancl. The fifth pancl displays ion temperature from the two detectors along with the electron temperature (solid line). Root mean square errors associated with 16 point ( 3 second) averages are shown in the bottom panel. 
1830 the $\phi$ angle remains similar to the IMF angle with a value less than $360^{\circ}$. By this time the Geotail solar magnetospheric $Z$ coordinate has increased by a few $\mathrm{R}_{\mathrm{E}}$ and the tail has probably rotated in the opposite sense due to the changing IMF $B_{y}$. Indeed the spacecraft subsequently sees only field directions characteristic of the northern half of the tail in the shaded regions which would not be easily distinguishable from the IMF direction.

The observations discussed above are in many respects consistent with Geotail moving back and forth between the magnetosheath and the magnetotail. However, at a location less than $12 R_{E}$ from the expected tail axis this is not where such crossings would normally be expected. There are also other features that distinguish the unshaded region from a normal magnetosheath. As noted above, the density is only about half that of the solar wind. Normally the magnetosheath density, after increasing on passing through the near-earth bow shock, has decreased to near its solar wind density at a distance of $134 \mathrm{R}_{\mathrm{E}}$, although gas

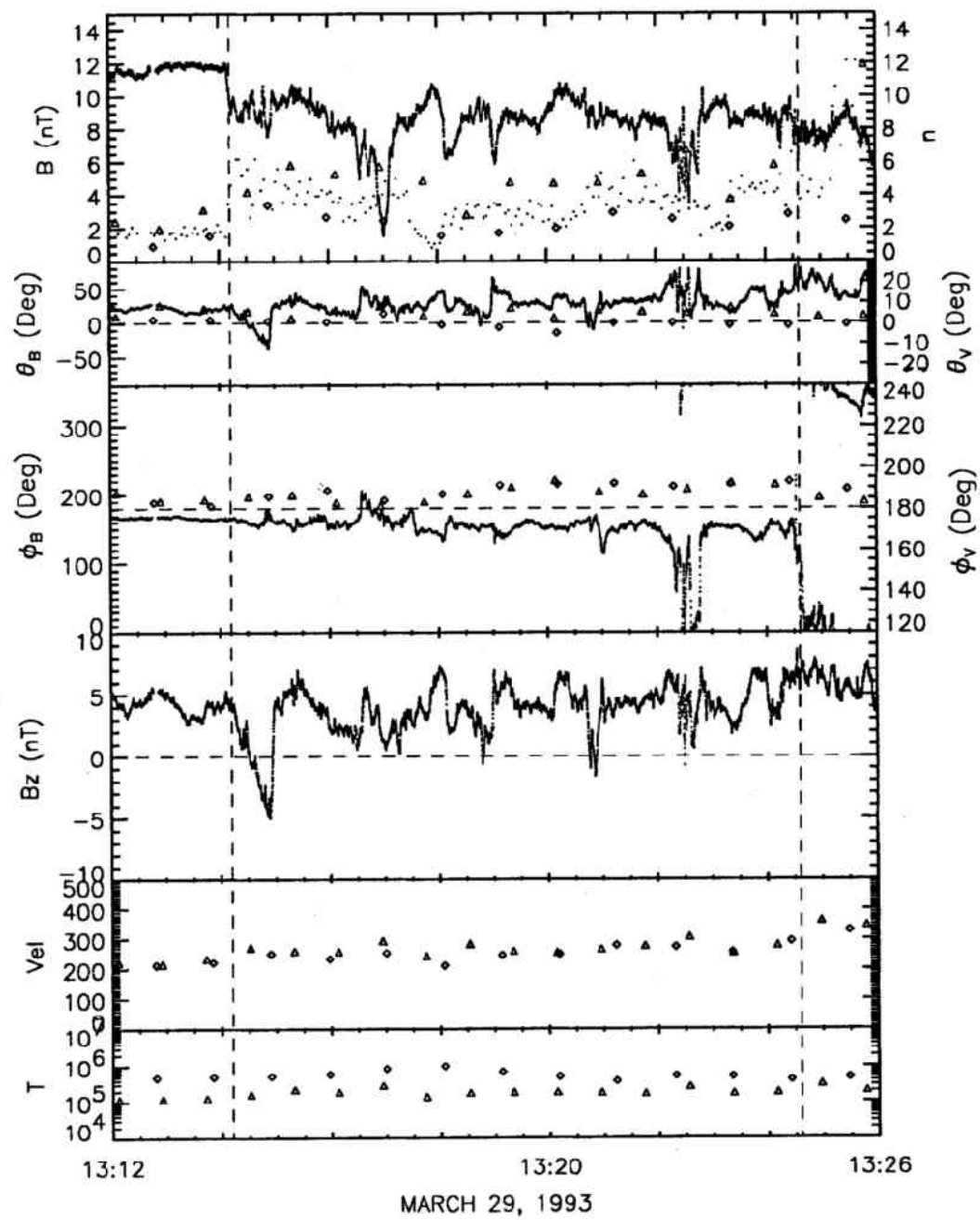

Fig. 2b. Fourteen minutes of highest resolution (16 measurements/s) magnetic field data are displayed with solid lines along with plasma data in a format similar to Fig. 2a. EFD densities are now represented by light points. The first vertical dashed line indicates the time when Geotail exited a low density, high field tail region and the second vertical dashed line indicates when the field longitude angle reverted to a direction characteristic of the exterior region. 
dynamic theory does indicate that the density along the stagnation stream line (i.e. near the boundary) can obtain values less than the solar wind (Siscoe et al., 1970). Note that the Geotail magnetic field in the unshaded regions also is lower than the IMF. A more unexpected feature of the unshaded region is the Geotail plasma flow direction shown in Fig. la. The north/south angle in the second panel (positive is northward) agrees well with the solar wind direction but the east/west angle (positive is dawnward) is dramatically different. Where the aberrated solar wind direction is about $2^{\circ}$ in the duskward direction, the average Geotail direction is about $6^{\circ}$ dawnward. This is not explained by simple models, but we note that an MHD simulation of an event on October 27, 1992, using measured IMF showed exterior flows in different directions in different locations that were more complex than what would be expected from a gas dynamic model (Frank et al., 1995). Nishida et al. (1995) have also proposed $B_{y}$-dependent cross tail flows that will be discussed below.

Since the terms magnetosheath and tail connote characteristics not necessarily seen in the Geotail data on March 29, we will refrain from using these terms. Still it does seem clear that Geotail is moving back and forth between a higher density, higher velocity region where the magnetic field direction resembles that of the IMF (i.e. a region similar to the magnetosheath) and another region of lower density and velocity where the field is more taillike (i.e. a region similar to the tail). In the following discussion we will refer to these two regions as the exterior and interior regions respectively.

Figure 2a illustrates Geotail plasma and higher resolution (3 second averages) magnetic field data. Plasma data from both the CPI solar wind and hot plasma instruments on Geotail are shown by solid triangles and open diamonds respectively. The solar wind instrument looks exclusively at 30 azimuthal angular intervals in the solar direction $\left( \pm 56^{\circ}\right)$ and hence is appropriate for accurately measuring the high velocity directed flows in the magnetosheath-like region. The hot plasma instrument covers $4 \pi$ steradians at lower angular resolution and hence is more appropriate for measuring, hotter, more isotropic plasma with arbitrary flow directions in the taillike regions. Both data sets are shown since the spacecraft moves between regions where one or the other is the appropriate measurement. The line in the top panel shows an estimate of the density obtained from the spacecraft potential measured by the Electric Field Detector (EFD) (Nihei, private communication) at a resolution of one measurement every spacecraft spin of 3 seconds duration. It can be noted in this and subsequent figures that this EFD density tends to agree with either the CPI solar wind density or the hot plasma density depending on which is the relevant instrument. The high time resolution of the EFD measurement provides an indication of the time scale of the more rapid density changes.

Figure 2a illustrates the interval from 1300 to 1400 hours where Geotail emerges from a relatively clear tail where plasma on taillike field lines with density of slightly greater than $1 / \mathrm{cc}$ is flowing tailward at a speed about two thirds the solar wind velocity. At 1314 UT, marked by a vertical dashed line, the field strength abruptly decreases and begins to fluctuate, even at higher frequencies as indicated by an increase in the three second rms values in the bottom panel. Also the density increases to about $4 / \mathrm{cc}$ and the velocity begins to increase. The average field direction, however, does not change significantly from its taillike orientation at this time. About 10 minutes later at 1326 the azimuthal field angle suddenly assumes the direction characteristic of the IMF and the velocity abruptly increases to approximately the solar wind velocity. Density varies between 5 and 10/cc for the rest of this hour. Large magnetic field directional fluctuations continue despite the fact that the IMF direction is very steady.

Figure $2 \mathrm{~b}$ shows data from 1312 to 1326 on an expanded time scale. The highest resolution magnetic field data (16 measurements/s) are shown by solid lines and the plasma data is repeated with the EFD density indicated by light dots. Note that the density change at $131415 \mathrm{~s}$ is on the same time scale as the magnetic ficld which is only a few seconds. The high frequency magnetic field fluctuations tend to be in the vicinity of the proton gyrofrequency.

Figure 3 shows data for the following hour of 1400-1500 in the same format as Fig. 2a. Comparison with Fig. 2a reveals that near 1400 the highly fluctuating magnetic field in the exterior region suddenly becomes much more stable. One might want to attribute this change to the spacecraft moving further away from the tail and the interaction region near a boundary, but at 1431 the spacecraft reenters the interior 


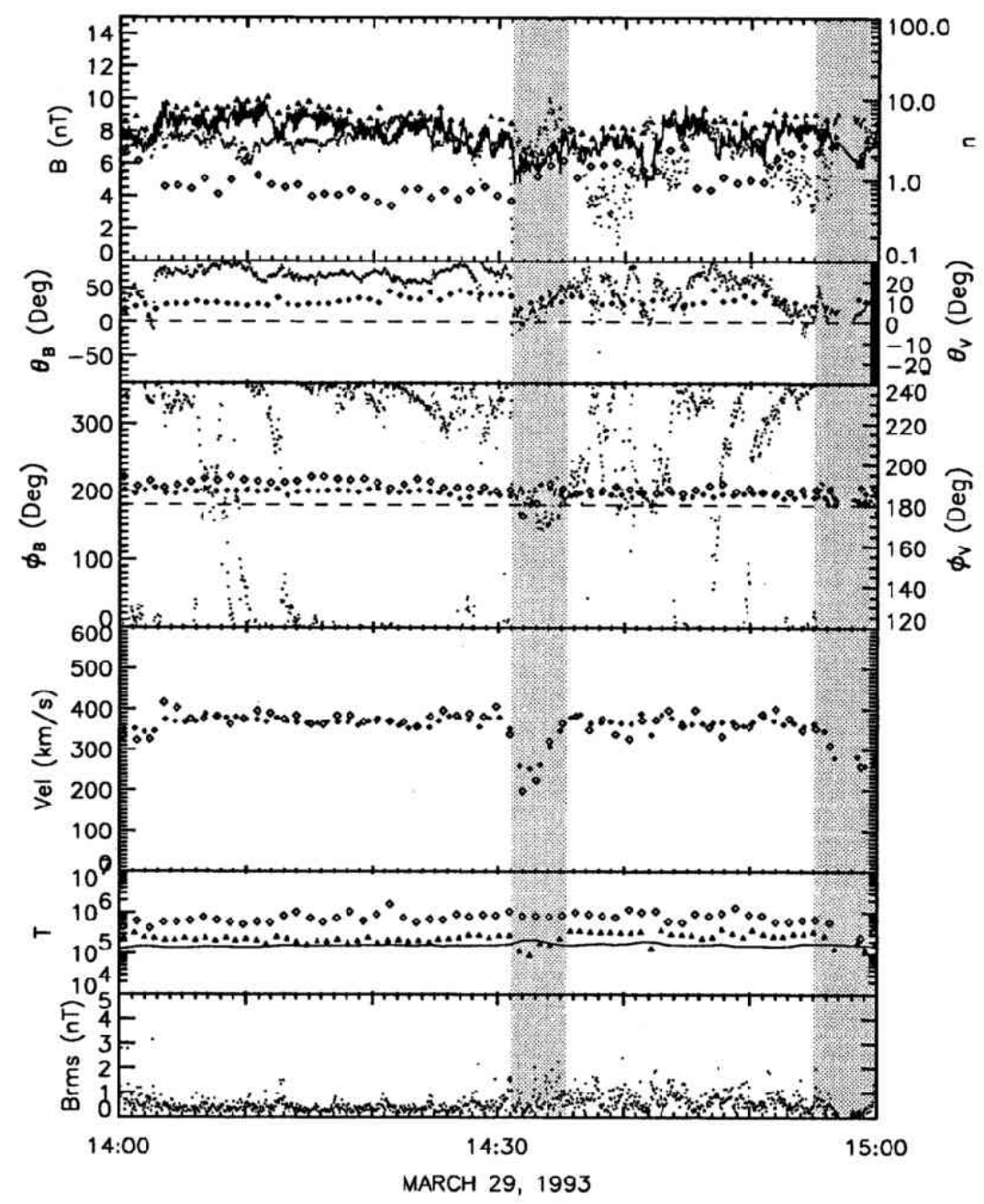

Fig. 3. One hour of data in the format of Fig. 2a. Magnetic fields in the exterior region are much quieter than those seen during the previous hour.

region without passing back through a region of fluctuations. Note that the flow direction in the exterior region is about $187^{\circ}\left(7^{\circ}\right.$ dawnward $)$ except that it decreases by about $2^{\circ}$ in the five minutes before the crossing, probably due to the approaching boundary. At this crossing the density and velocity change abruptly to lower values and the field direction again assumes the direction characteristic of the southern hemisphere of the tail. The spacecraft again crosses back to the exterior region some 4 to 6 minutes later, but this transition appears very different with the field direction and velocity making the transition very gradually. Crossings with such very different character are seen throughout this day and make generalizations difficult. Note that the magnetic field on returning to the exterior region at 1435 is quite disturbed in contrast to the field seen before entering the interior region. Another transition back to the interior region occurs at 1456 which is again relatively abrupt.

The following hour of $1500-1600$ is illustrated by Fig. 4a. Geotail remains in the interior region at the beginning of this hour as indicated by the southern hemisphere field direction. At higher resolution Fig. $4 \mathrm{~b}$ indicates this region of southern field direction from $145510 \mathrm{~s}$ to $151030 \mathrm{~s}$ bracketed by vertical 


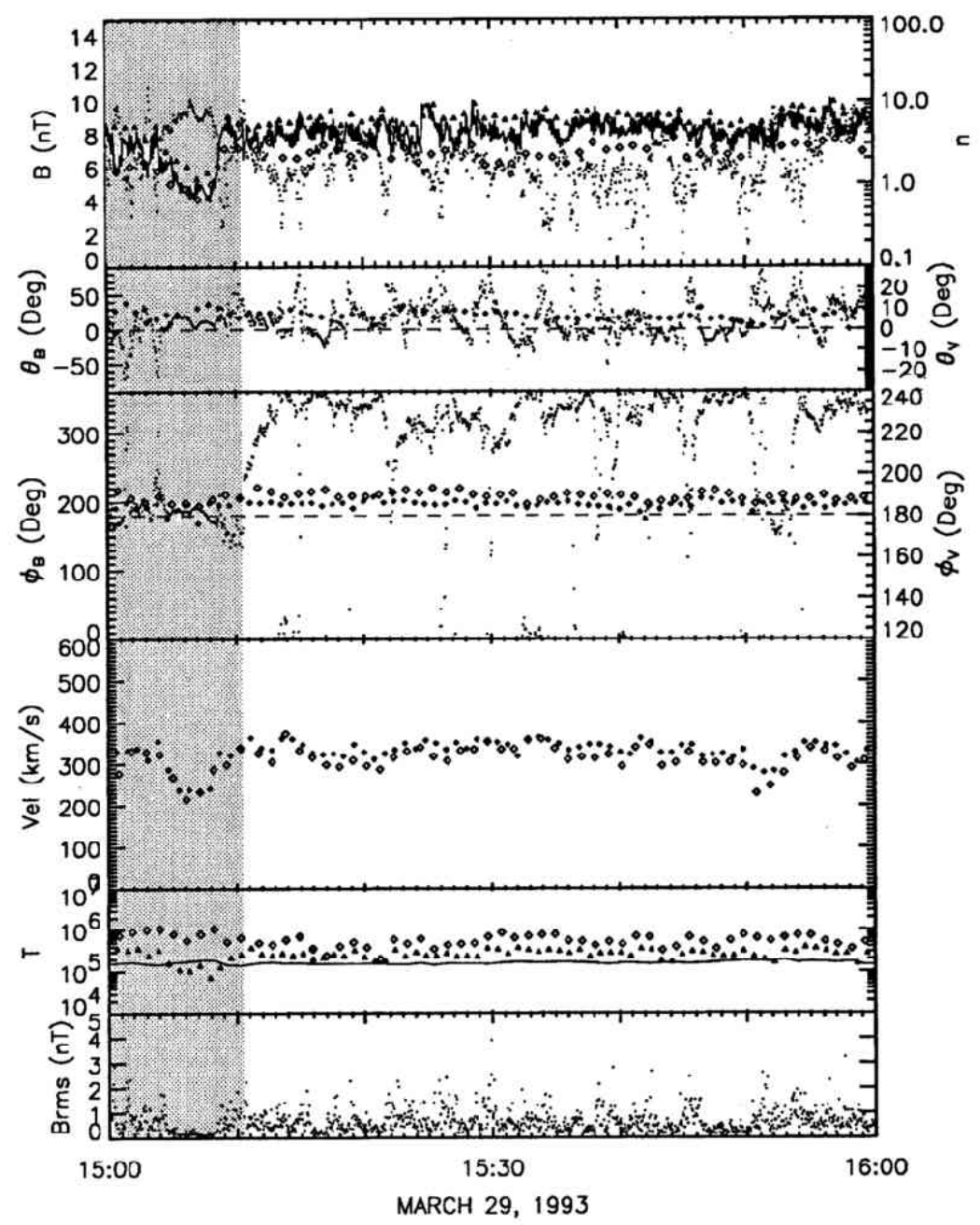

Fig. 4a. One hour of data in the format of Fig. 2a. The magnetic field data in the exterior (unshaded) region is highly variable despite the steadiness of the IMF.

dashed lines, but lower field strengths and higher densities and velocities extend into the southern hemisphere field region for about a minute at each crossing. Intervals of enhanced density centered at 1501 $30 \mathrm{~s}$ and 1504 suggest additional approaches to the boundary, but this does not necessarily explain the intervals of strongly southward field at these times. Figure 4 a shows that when Geotail returned to the exterior region after 1510, the magnetic field was highly disturbed for the rest of the hour with large peaks in the latitude angle recurring every few minutes. Although there are occasional brief returns to orientations more similar to the southern tail, they are not associated with any tendency for the density or velocity to decrease.

Figure 5 includes complex intervals of the interior region in high resolution. Shading indicates two intervals of low density and velocity. Two clear intervals characteristic of the southern tail, although with large $B_{z}$, occur from 1714 to $171715 \mathrm{~s}$ and from 1719 to $172015 \mathrm{~s}$. Low density and velocity persist in the intervening interval of almost two minutes duration which is characterized by a large and variable southward field. This region can be identified as the plasma sheet, or perhaps a flux rope. Another clear 


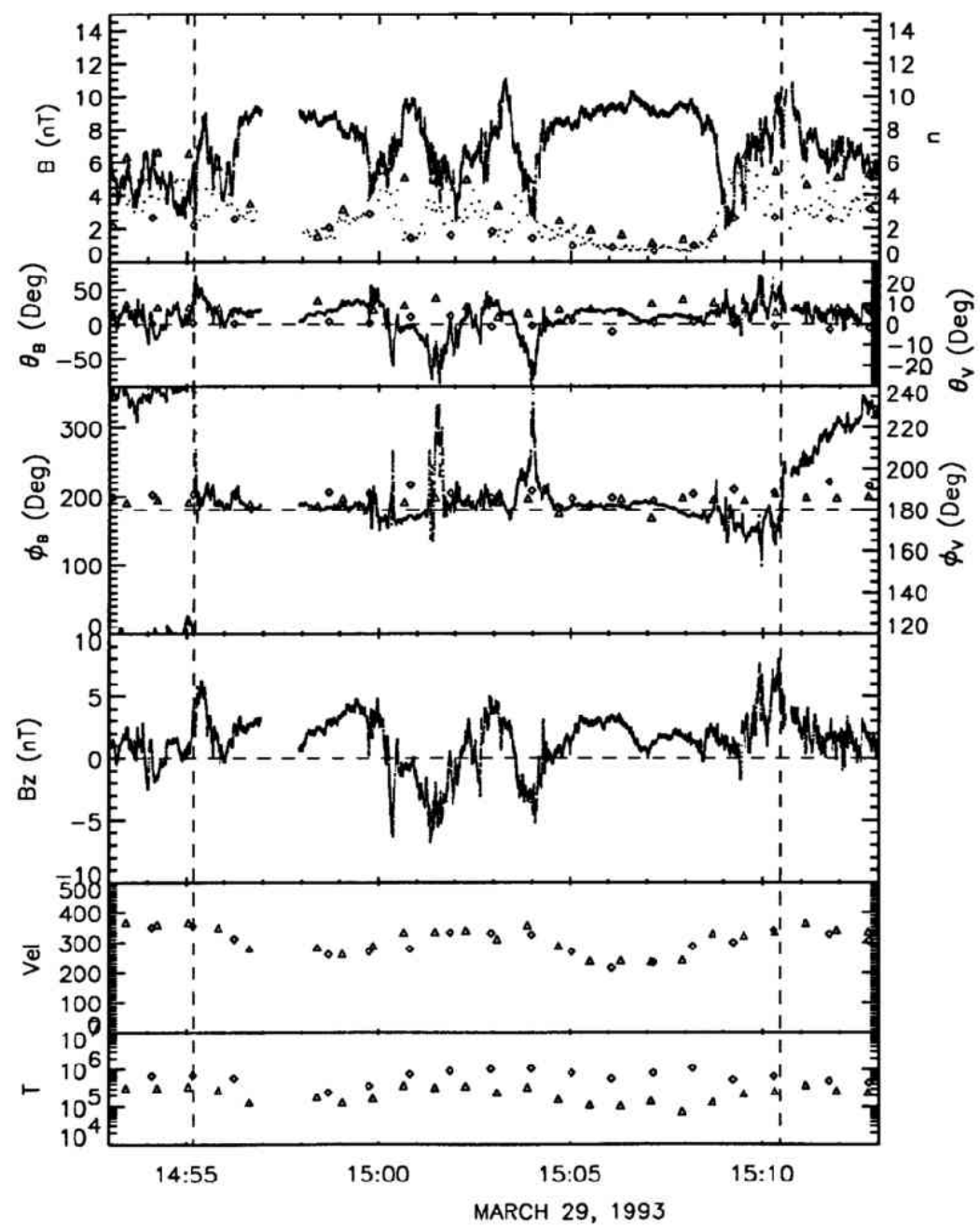

Fig. 4b. Twenty minutes of high resolution magnetic field data in the format of Fig. $2 b$ for the interior interval spanning an interval of Figs. 3a and 4a. Vertical dashed lines indicate when the longitude angle changes from or to its exterior orientation.

interval in the interior region occurs from $172435 \mathrm{~s}$ to $172745 \mathrm{~s}$. The latitude angle shows a south to north variation throughout this interval which is a similar tendency to the previous intervals.

As a final example we present in Fig. 6 the high resolution data for a pair of crossings between the northern hemisphere internal region and the exterior region. Shading delineates the low density, high field region with the boundaries chosen as decreases in the high field strengths. Since the longitude angle of the internal field is now about the same as the external field there is a smaller angular discontinuity to delineate any magnetic field boundary. The boundaries could well have been moved out to modest angular changes at $202440 \mathrm{~s}$ and $202945 \mathrm{~s}$ in which case one could claim that weaker field and higher densities exist inside the boundary as often happened on crossings involving the southern hemisphere. The waves with periods of about $8 \mathrm{~s}$ seen in the $B_{z}$ component in the low density region are left handed, primarily transverse waves near the proton gyrofrequency. Similar waves are seen before the density decrease at about twice the frequency. 


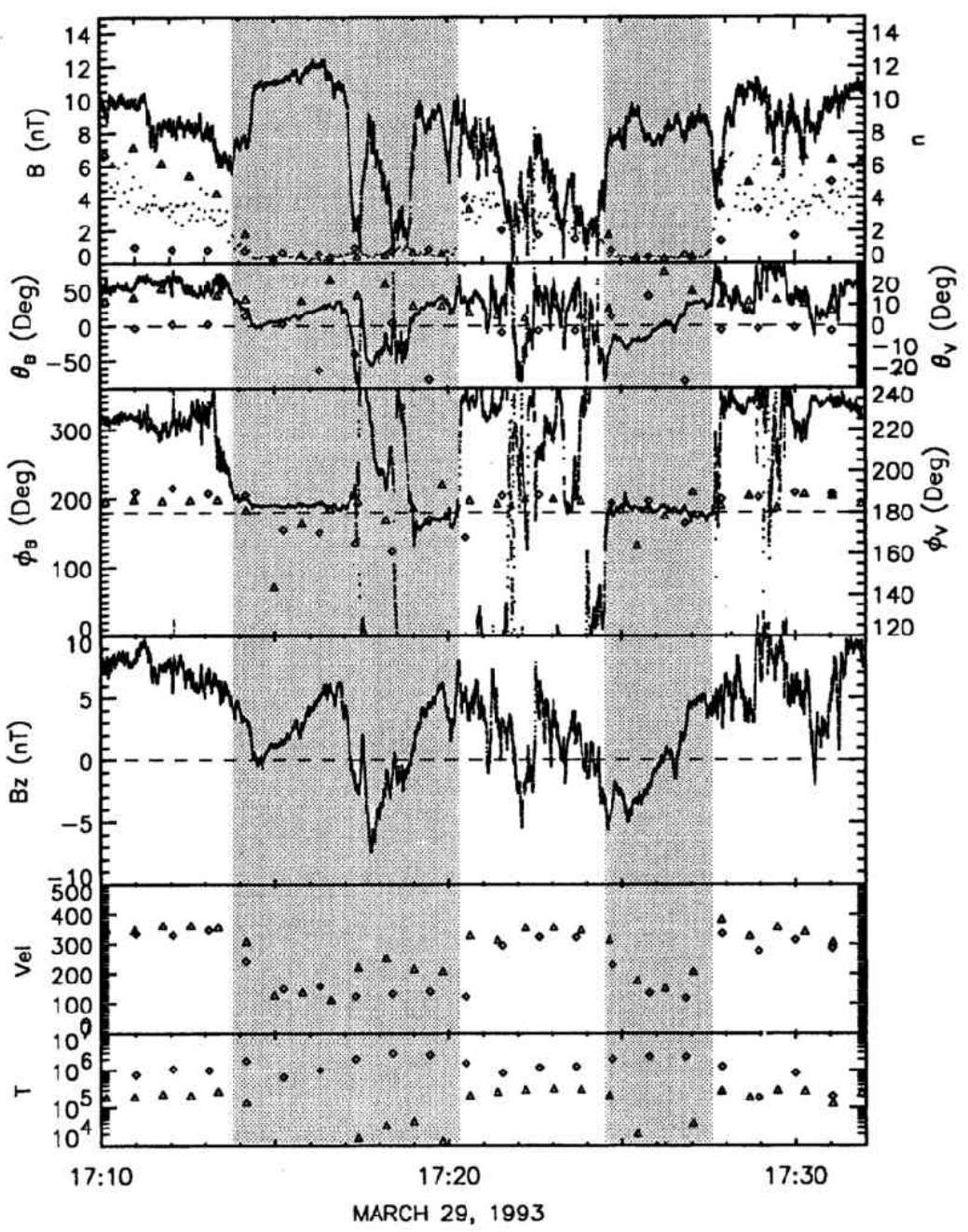

Fig. 5. High resolution magnetic field data in the format of Fig. $2 \mathrm{~b}$ showing two examples of the interior region, the first of which contains plasma sheet.

\section{Boundary Normals}

Although the character of the crossings between the exterior and interior regions varies considerably, in many cases it is possible to use the minimum variance technique (Sonneup and Cahill, 1967) to determine a boundary normal. This has been possible in 10 inbound (i.e., exterior to interior crossings) and 12 outbound cases. The first 13 crossings which occurred prior to 1830 UT involved crossings from southern hemisphere $\left(B_{x}<0 \mathrm{nT}\right)$ tail field lines and hence had relatively large angular changes due to the positive $B_{x}$ in the exterior region; the next 9 crossings involved transitions from northern hemisphere field lines and smaller angular changes. There were no notable differences in these two sets of normals. (There is not a one to one correspondence between the boundaries where normals were calculated and the boundaries of the shaded regions in Fig. 1 due to the fact that (1) normals could not be calculated at all the boundaries in Fig. 1 and (2) some multiple crossings were too closely spaced to be resolved in Fig. 1.) 


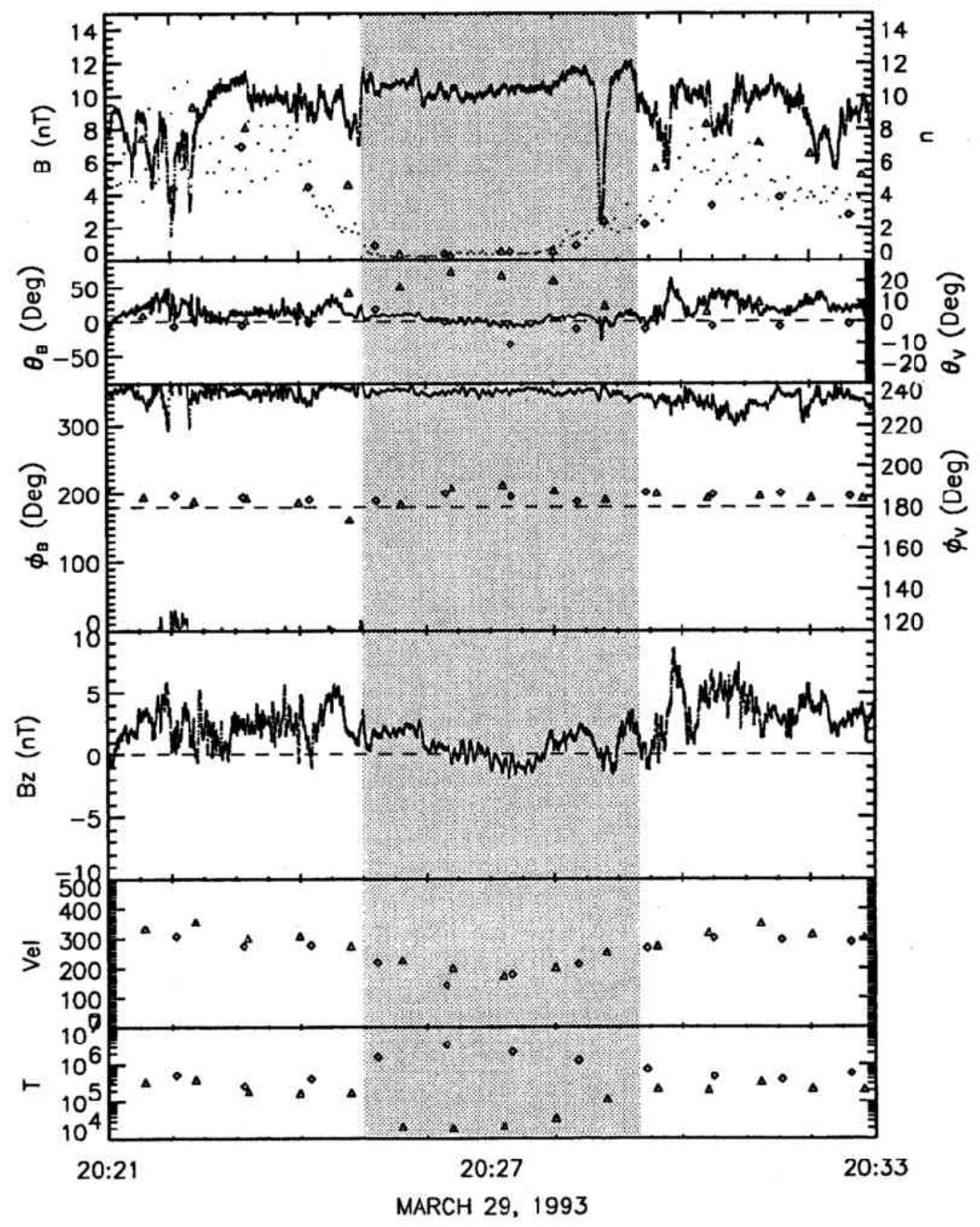

Fig. 6. High resolution measurements of a northern hemisphere, low density internal region when the exterior region had a magnetic field with a similar orientation to the internal field.

The inbound and outbound normals are plotted separately in Fig. 7. The figure shows the boundary normals projected on the solar magnetospheric $x y$ and $y z$ planes. If the component out of the plane of the figure were zero, each line would reach the unit circle, so the length of the line provides a graphic indication of how near the normal is to the plane of the figure. In almost all cases the $Y$ component is the dominant component as would be expected for an equatorial location and a normal to a cylindrical tail. Figures $7 \mathrm{c}$ and $7 \mathrm{~d}$ indicate that the normals are scattered rather uniformly in the north/south direction but Figs. $7 \mathrm{a}$ and $7 \mathrm{~b}$ show that the outbound normals are almost all tilted toward the sun (by an average of $13.4^{\circ}$ ) whereas the inbound normals, on average, are nearly perpendicular to the $X$ axis (average $0.3^{\circ}$ ).

This tendency for outbound normals to be tilted more toward the sun than inbound normals is consistent with tailward propagating structures on the boundary and has been found in many studies (i.e., Chen et al., 1993; Nishida et al., 1995). The fact that the overall average also is tilted toward the sun is consistent with flaring of the tail, but, as Chen and Kivelson (1993) point out, a non-sinusoidal wave can give enhanced outbound tilts so this positive tilt is not necessary evidence for flaring. 

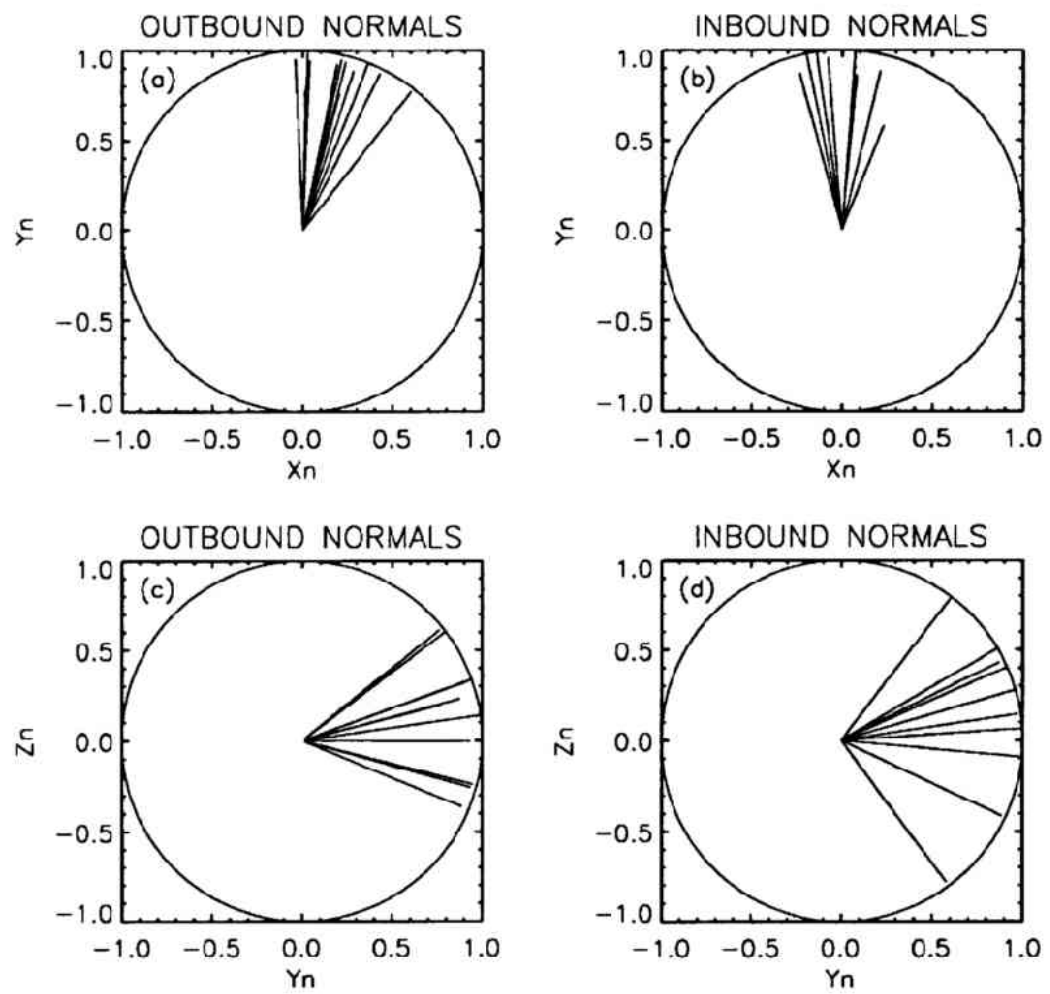

Fig. 7. Inbound and outbound normals are plotted in the $x y$ plane (a) and (b) and the $y z$ plane (c) and (d). Outbound normals (a) are tilted more toward the sun than inbound normals (b).

\section{Summary and Discussion}

The recurring observation of cool, dense, tailward-flowing plasma on non-taillike magnetic field lines $134 R_{E}$ behind the earth at a location only $12 R_{E}$ from the tail axis on March 29, 1993, implies the existence of a geomagnetic tail that is very different from a nominal or average tail. Figure 8 illustrates the equatorial plane view of such a tail (solid line) relative to an average tail (dashed line). The solid line magnetotail is drawn through the Geotail position $11.5 \mathrm{R}_{\mathrm{E}}$ from the tail axis. The insert illustrates wavey structures on the boundary that may be producing magnetopause normals that are found to tilt more toward the sun on exits from the interior region than those on entries.

The fact that these boundary observations occurred under unusually steady and northward IMF conditions supports similar observations with the ISEE 3 spacecraft (Fairfield, 1993) and are highly suggestive that the northward IMF is responsible for the unusual tail. The observations are consistent with recent global simulations for directly northward IMF which indicates that the radius of the distant tail becomes small (Ogino et al., 1992, 1994; Usadi et al., 1993; Raeder et al., 1995) or even zero (Fedder and Lyon, 1995).

In spite of the general agreement with simulations, the interpretation of the detailed Geotail observations is not straightforward. The fact that the Geotail plasma flow in the exterior region was directed $6^{\circ}$ dawnward while the solar wind flowed $2^{\circ}$ duskward is unexpected. If such flow were tangent to a boundary that was decreasing in radius with distance down the tail, the rate of decrease would be occurring at a rate of $1.4 \mathrm{R}_{\mathrm{E}}$ every $10 \mathrm{R}_{\mathrm{E}}$ down the tail $\left(10 \tan 8^{\circ}\right)$. In the unlikely event of this rate persisting over great distances, the tail would close at $220 \mathrm{R}_{\mathrm{E}}$. Although this is possible, it seems unlikely, particularly since the IMF is not directly northward as in the simulations that indicate a closed tail. 


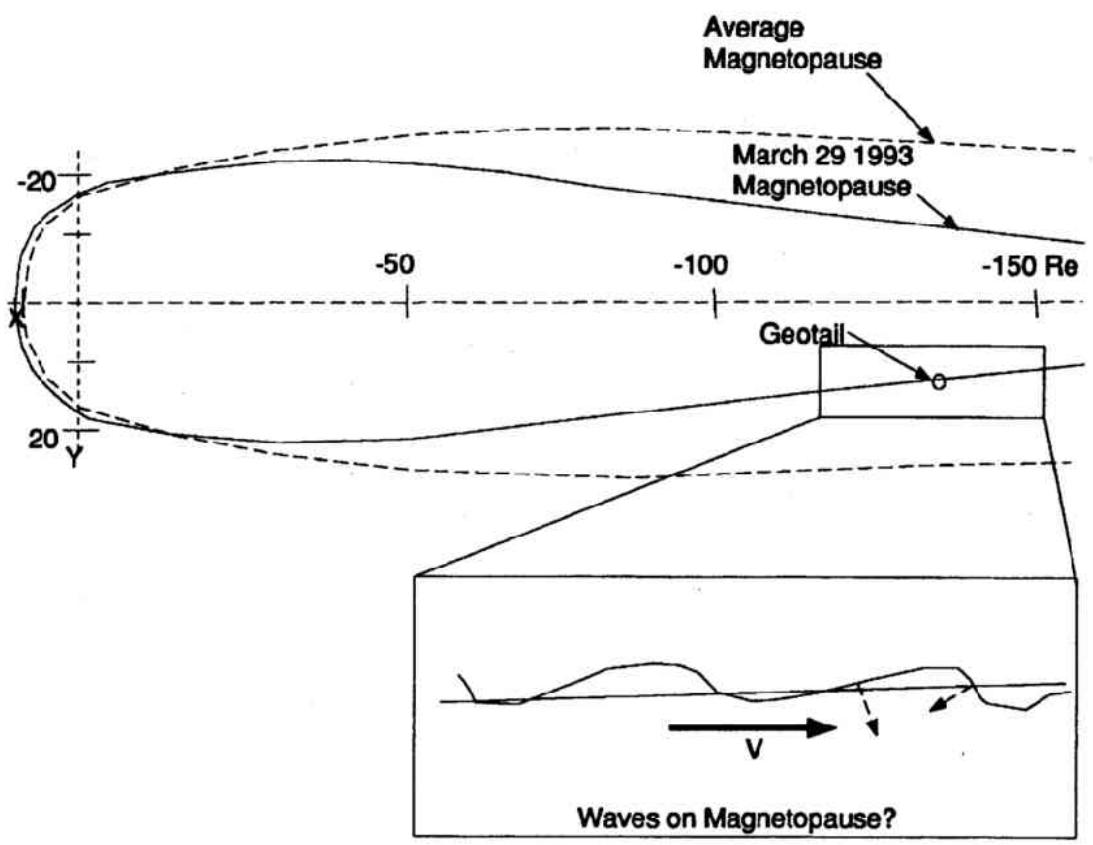

Fig. 8. An average tail (dashed line) is compared to the tail necessary to explain the presence of Geotail in a dense, flowing magnetosheath-like plasma with magnetic field similar to the IMF.

Another possibility is that there is flow through the boundary as seen by Gosling et al. (1984), Mozer et al. (1994) and deduced by Yamamoto et al. (1994). Such direct entry is also supported by high resolution measurements near the boundaries. At roughly half the boundaries, the inner region exhibits a decrease in ficld strength and an increase in density before the field direction transition. Figure $2 \mathrm{~b}$ where the initial change takes place at $131425 \mathrm{~s}$ but the direction change occurs 10 min later is an extreme example; in most cases the directional change occurs within a minute of the field strength/density change. These changes along with the flowing nature of this internal plasma are consistent with direct entry of the plasma.

Such flow into the tail may also be consistent with an innovative suggestion that has been proposed to interpret cross-tail flow observations seen by Nishida et al. (1995). These authors suggest cross-tail convection is parallel to the equatorial current sheet of a tail that has been twisted by a positive IMF $B_{y}$. For this positive $B_{y}$ the component of $V$ perpendicular to $B$ is duskward and northward on northern hemisphere field lines and southward and dawnward on southern hemisphere field lines; everything reverses for negative IMF $B_{y}$. For our case the small IMF $B_{y}$ should produce a modest counterclockwise (looking downtail from earth) twist of the tail which is consistent with observations of the southern hemisphere prior to 1830 UT. The dawnward flow and northward flow in the southern hemisphere are also in agreement with the model. Later when the IMF $B_{y}$ has reversed sign, observations of the continuing dawnward and northward convection, but in the northern tail, are again in agreement with the model. However the fact that the IMF changes very slowly and $B_{y}$ is near zero for several hours while the flow is continually dawnward and northward for the whole interval casts some doubt on this interpretation. Another similar northward field event with larger and more variable $B_{y}$ occurred on July 7, 1993, but was not discussed in this paper. There a large change in the Geotail flow was associated with an IMF change to a negative $B_{y}$. The Geotail flow in the northern hemisphere was dawnward in agreement with the model, but southward, which is contrary to the model.

Boundary normals calculated in this study tended to be tilted slightly toward the sun for outbound crossings but perpendicular to the earth-sun line for inbound crossings, a fact that is consistent with earlier 
conclusions about tailward propagating structures on the boundary (Chen et al., 1993; Chen and Kivelson, 1993; Nishida et al., 1995). Chen and co-authors used two closely spaced satellites to argue that the waves they observed on the boundary were non-sinusodal, with outbound normals tilted more in the sunward direction than inbound normals were tilted away. This situation is illustrated by the insert in Fig. 8 . If this effect is controlling the Geotail measurements, the fact that the average of all Geotail measurements was tilted toward the sun may not mean the boundary is flaring. Although Geotail measurements did not show the obvious periodicity of crossings as observed nearer the earth, there were intervals of similar several minute periodicities in magnetic field fluctuations in the exterior region (i.e., 1510-1600 in Fig. 4a). These fluctuations occurred without decreases to the lower densities and velocities of the interior region but could perhaps indicate approaches to the outer part of the inner region.

\section{Conclusions}

The frequent observation of magnetosheath-like magnetic fields and plasmas only $12 R_{E}$ from the tail axis on March 29, 1993, provides further evidence that the magnetotail assumes a very different configuration on this day with large steady northward IMF. These data, along with the numerous global simulations cited above and the well-known diminution of polar cap size with northward IMF, all provide compelling evidence for a magnetosphere with more field lines closing close to the earth and fewer forming a broad extended geomagnetic tail during these rare intervals of strong northward field. It seems likely that high latitude reconnection between the IMF and field lines poleward of the dayside cusp are involved in producing this unusual tail (e.g., Song and Russell, 1992; Crooker, 1992).

The observation of flows tilted $8^{\circ}$ toward the tail axis rather than parallel to a cylindrical tail most likely results from a combination of flow along a boundary of diminishing radius and flow across the boundary into the tail. Although the observed average tilt of boundary normals in the sunward direction might normally be taken as evidence for a flaring tail rather than a tail of diminishing radius, nonsinusoidal waves (Chen and Kivelson, 1993; Chen et al., 1993) or similar structures on the boundary may explain these tilts and be consistent with the diminishing radius. Further interpretation may have to await simulations for these particular conditions such as have been carried out to explain boundary positions and movements on another day (Frank et al., 1995).

We thank the MIT Solar Wind group for the use of the IMP 8 solar wind data.

\section{REFERENCES}

Chen, S. H. and M. G. Kivelson, On non-sinusoidal waves at the earth's magnetopause, Geophys. Res. Lett., 20,2699-2702, 1993.

Chen, S. H., M. G. Kivelson, J. T. Gosling, R. J. Walker, and A. J. Lazarus, Anomalous aspects of magnetosheath flow and of the shape and oscillations of the magnetopause during an interval of strongly northwardinterplanetary magnetic field,J. Geophys. Res., 98, 5727-5742, 1993.

Cowley, S. W. H., Magnetospheric asymmetries associated with the $Y$-component of the IMF, Planet. Space Sci., 29, 79-96, 1981.

Crooker, N. U., Reverse convection, J. Geophys. Res., 97, 19,363-19,372, 1992.

Fairfield, D. H., On the structure of the distant magnetotail: ISEE 3, J. Geophys. Res., 97, 1403-1410, 1992.

Fairfield, D. H., Solar wind control of the distant magnetotail: ISEE 3, J. Geophys. Res., 98, 21,265-21,276, 1993.

Fedder, J. A. and J. G. Lyon, The Earth's magnetosphere is $165 \mathrm{R}_{\mathrm{E}}$ long: Self-consistent currents, convection, magnetospheric structure and processes for northward interplanetary magnetic field, J. Geophys. Res., 100, 3623-3635, 1995.

Frank, L. A., M. Ashour-Abdalla, J. Berchem, J. Raeder, W. R. Paterson, S. Kokubun, T. Yamamoto, R. P. Lepping, F. V. Loroniti, D. H. Fairfield, and K. L. Ackerson, Observations of plasmas and magnetic fields in Earth's distant magnetotail: Comparison with a global MHD model, J. Geophys. Res., 100, 19,177-19,190, 1995.

Gosling, J. T., D. N. Baker, S. J. Bame, E. W. Hones, D. J. McComas, R. D. Zwickl, J. A. Slavin, E. J. Smith, and B. T. Tsurutani, Plasma entry into the distant tail lobes: ISEE-3, Geophys. Res. Lett., 11, 1078-1081, 1984.

Mozer, F. S., H. Hayakawa, S. Kokubun, M. Nakamura, T. Okada, T. Yamamoto, and K. Tsuruda, Direct entry of dense flowing plasmas into the distant tail lobes, Geophys. Res. Lett., 21, 2959-2962, 1994.

Nishida, A., T. Mukai, T. Yamamoto, Y. Saito, S. Kokubun, and K. Maezawa, GEOTAIL observations of magnetospheric convection in the distant tail at $200 \mathrm{R}_{\mathrm{E}}$ in quiet times, J. Geophys. Res., 100, 1995 (in press). 
Ogino, T., R. J. Walker, and M. Ashour-Abdalla, A global magnetohydrodynamic simulation of the magnetosheath and magnetosphere when interplanetary magnetic field is northward, IEEE Trans. Plasma Sci., 20, 817-828, 1992.

Ogino, T., R. J. Walker, and M. Ashour-Abdalla, A global magnetohydrodynamic simulation of the response of the magnetosphere to a northward turning of the interplanetary magnetic field, J. Geophys. Res., 99, 11,027-11,042, 1994.

Owen, C. J., J. A. Slavin, I. G. Richardson, N. Murphy, and R. J. Hynds, Average motion, structure, and orientation of the distant magnetotail determined from remote sensing of the edge of the plasma sheet boundary layer with $E>35 \mathrm{keV}$ ions, J. Geophys. Res., 100, 185-204, 1995.

Raeder, J., R. J. Walker, and M Ashour-Abdalla, The structure of the distant geomagnetic tail during long periods of northward IMF, Geophys. Res. Lett., 22, 349-352, 1995.

Sibeck, D. G., J. A. Slavin, E. J. Smith, and B. T. Tsurutani, Twisting of the geomagnetic tail, in Solar Wind Magnetosphere Coupling, edited by Y. Kamide and J. A. Slavin, pp. 731, Terra Scientific, Tokyo, 1986.

Siscoe, G. L., F. L. Scarf, D. S. Intriligator, J. H. Wolfe, J. H. Binsack, H. S. Bridge, and V. M. Vasyliunas, Evidence for a geomagnetic wake at 500 Earth radii, J. Geophys. Res., 75, 5319-5330, 1970.

Song, P. and C. T. Russell, Model of the formation of the low-latitude boundary layer for strongly northward interplanetary magnetic field, J. Geophys. Res., 97, 1411-1420, 1992.

Sonnerup, B. U. O. and L. J. Cahill, Magnetopause structure and attitude from Explorer 12 observations, J. Geophys. Res., 72, 171-183, 1967.

Usadi, A., A. Kageyama, K. Watanabe, and T. Sato, A global simulation of the magnetosphere with a long tail: Southward and northward interplanetary magnetic field, J. Geophys. Res., 98, 7503-7517, 1993.

Yamamoto, T., K. Shiokawa, and S. Kokubun, Dense plasmas in the distant magnetotail as observed by Geotail, Geophys. Res. Lett., 21, 2879-2882, 1994. 